\title{
Identification and characterization of Thermus thermo- philus HB8 RuvA protein, the subunit of the RuvAB protein complex that promotes branch migration of Holliday junctions
}

\author{
Takayuki Ohnishi ${ }^{1}$, Hiroshi Iwasaki ${ }^{1,2, *}$, Yoshizumi Ishino ${ }^{3}$, Seiki Kuramitsu ${ }^{4}$, \\ Atsuo Nakata ${ }^{5}$, and Hideo Shinagawa ${ }^{1 *}$. \\ ${ }^{1}$ Department of Molecular Microbiology, Research Institute for Microbial Diseases, Osaka \\ University, Suita, Osaka 565-0871, Japan \\ ${ }^{2}$ PRESTO, JST, Suita, Osaka 565-0871, Japan \\ ${ }^{3}$ Department of Molecular Biology, Biomolecular Engineering Research Institute, \\ Suita, Osaka 565-0874, Japan \\ ${ }^{4}$ Department of Biology, Graduate School of Science, Osaka University, \\ Toyonaka, Osaka 560-0043, Japan \\ ${ }^{5}$ Department of Marine Biotechnology, Faculty of Technology, \\ Fukuyama University, Fukuyama, \\ Hiroshima 729-0292, Japan
}

(Received 25 September 2000, accepted 16 October 2000)

\begin{abstract}
The Escherichia coli ruvA and ruvB genes constitute an SOS-regulated operon. The products of these genes form a protein complex that promotes branch migration of the Holliday junction, an intermediate of homologous recombination. RuvA protein binds specifically to the Holliday junction and recruits RuvB protein to the junction. RuvB is an ATP-driven motor protein involved in branch migration. We previously cloned the $\operatorname{ruvB}$ gene of the thermophilic bacterium Thermus thermophilus HB8 (Tth) and found that, in contrast to the operon structure in most mesothermic bacteria, the ruvA gene is absent from the vicinity of ruvB. In this work, we cloned the ruvA gene from T. thermophilus HB8 and analyzed its nucleotide sequence. Tth RuvA is a protein of 20,414 Da consisting of 191 amino acid residues, and is $37 \%$ identical in amino acid sequence to $E$. coli RuvA. Tth ruvA complemented the DNA repair defect of E. coli $\Delta r u v A$ mutants. The purified Tth RuvA protein stimulated Tth RuvB activities, such as hydrolysis of ATP and promotion of branch migration of the Holliday junction, in a manner similar to the RuvA-RuvB interactions observed in E. coli. In addition, Tth RuvA stimulated the $E$. coli RuvB activities in vitro, which was well consistent with the results of in vivo hetero-complementation experiments.
\end{abstract}

\section{INTRODUCTION}

Homologous recombination is an essential biological process that generates genetic diversity and is necessary to repair damaged DNA. The pathways of homologous recombination have been extensively studied in Escherichia coli. The Holliday structure, which is a crucial recombination intermediate, is formed through homologous pairing and strand exchange reactions mediated by RecA protein and its accessory proteins (Kowalczykowski

Edited by Eiichi Ohtsubo

* Corresponding authors. E-mail: shinagaw@biken.osaka-u.ac.jp or iwasaki@biken.osaka-u.ac.jp et al., 1994). The RuvABC proteins are involved in processing Holliday junctions during the late stage of homologous recombination in E. coli (Shinagawa and Iwasaki, 1996; West, 1997). The RuvAB protein complex promotes branch migration of Holliday junctions (Iwasaki et al., 1992; Tsaneva et al., 1992), and RuvC resolves the junction when it encounters favorable Holliday junction sequences (Iwasaki et al., 1991; Shah et al., 1994). The $22-\mathrm{kDa}$ RuvA protein forms a stable tetramer, binds specifically to the junction, and facilitates the loading of RuvB to the junction (Iwasaki et al., 1992; Parsons et al., 1992). The $37-\mathrm{kDa}$ RuvB protein forms a hexameric ring and is a motor protein involved in branch migration that requires ATP hydrolysis (Shiba et al., 1993; Stasiak et al., 1994). 
RuvB has intrinsic ATPase activity, which is enhanced by duplex DNA and RuvA in a synergistic manner (Iwasaki et al., 1989; Shiba et al., 1991). The 19-kDa RuvC protein forms a dimeric endonuclease, specifically binds to the Holliday junction, and resolves it by introducing two nicks at symmetrical positions across the junction (Dunderdale et al., 1991; Iwasaki et al., 1991; Shida et al., 1996).

RuvA and RuvB orthologs are highly conserved among bacteria. In $E$. coli the $r u v A$ and $r u v B$ genes constitute a single operon regulated by the SOS system (Benson et al., 1988; Shinagawa et al., 1988). In contrast, ruvC belongs to a separate non-SOS operon that is located immediately upstream of ruvAB (Sharples and Lloyd, 1991; Takahagi et al., 1991). In many bacteria, such as Pseudomonas aeruginosa, Haemophilus influenzae Rd, and Mycobacterium leprae, ruv $C, \operatorname{ruv} A$, and $\operatorname{ruv} B$ constitute a single operon with the genes in this order (Hishida et al., 1996; Sharples et al., 1999). However, the ruvA gene of Thermus thermophilus HB8 (Tth) and two other thermophilic bacteria in which $r u v B$ genes were previously cloned and analyzed was not found in the vicinity of the $r u v B$ gene (Tong and Wetmur, 1996; Yamada et al., 1999). Although the Tth ruvB gene does not complement the UV sensitivity of the $E$. coli ruvB deletion strain, Tth RuvBmediated branch migration of Holliday junctions is stimulated by E. coli RuvA in vitro (Yamada et al., 1999). This suggested the existence of a RuvA ortholog in T. thermophilus HB8.

In the present study we cloned the ruvA gene of $T$. thermophilus HB8 and analyzed its sequence. We constructed an overproduction system for Tth RuvA in E. coli, purified the Tth RuvA protein, and analyzed its biochemical properties in vitro. Tth RuvA had similar properties to $E$. coli RuvA and interacted functionally with $E$. coli RuvB both in vivo and in vitro.

\section{MATERIALS AND METHODS}

E. coli strains and plasmids. HRS2300 ( $\Delta r u v$

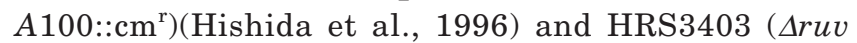
$A B 200: \mathrm{km}^{\mathrm{r}}$ ) (Ishioka et al., 1998), both derived from AB1157 (Bachmann, 1972), were used as host strains for UV sensitivity tests. HRS4000 ( $\triangle r u v A B C 200:: \mathrm{km}^{\mathrm{r}}$ ) (Yamada et al., 1999), derived from BL21(DE3) (Studier et al., 1990), was used for overexpression of the Tth RuvA protein. Plasmids used in this study are listed in Table 1.

Cloning and sequencing the Tth ruvA gene. Since a portion of a coding region of a RuvA-like protein was found from the Tth genome sequencing project, two primers based on the sequence of that region (F1: 5'-AACCTCGCCCTCTTTGAACTC-3' and R1: 5'-CTTTAGGGCCTCCTTGATGAG-3') were synthesized. These primers were subsequently used to amplify the Tth ruvA gene segment. The amplified DNA fragment $(\sim 400 \mathrm{bp})$ was used as a hybridization probe for the cloning of ruvA. The Tth genomic DNA was digested with $\mathrm{BamHI}$ and fractionated by agarose gel electrophoresis. A 5.5-kb DNA band that hybridized with the probe was isolated and ligated to BamHI-digested pUC19. Positive clones were identified by hybridization. One of these clones was named

Table 1. Plasmid list

\begin{tabular}{|c|c|c|}
\hline Plasmid & Characteristics & Source / reference \\
\hline pUC19 & A cloning vector & Laboratory stock \\
\hline pAF101 & A T7 expression vector with a pUC19 backbone & (Yamada et al., 1999) \\
\hline pAF104 & $\begin{array}{l}\text { A T7 expression vector derived from pAF101 carrying } \\
\text { lacI }{ }^{\mathrm{q}}\end{array}$ & This study \\
\hline pAF134 & pAF104 derivative carrying the $E$. coli ruv $A^{+}$gene & (Nishino et al., 1998) \\
\hline pBAD24 & An arabinose-inducible expression vector & (Guzman et al., 1995) \\
\hline pBluescript II SK (+) & A cloning vector & Laboratory stock \\
\hline pLysS & $\begin{array}{l}\text { Used for } \mathrm{T} 7 \text { expression system to reduce the basal level } \\
\text { expression }\end{array}$ & (Studier et al., 1990) \\
\hline pSTV28 & $\begin{array}{l}\text { pACYC184 derivative cloning vector compatible with } \\
\text { pUC-type plasmids }\end{array}$ & Takara-Shuzo (Kyoto) \\
\hline pTF100 & $\begin{array}{l}\text { pBAD24 derivative plasmid in which unique } N d e \mathrm{I} \text { and } \\
\text { BglII sites were created downstream of the ParaC } \\
\text { promoter }\end{array}$ & This study \\
\hline pTRA104 & $\begin{array}{l}\text { pUC19 derivative carrying } 5.5-\mathrm{kb} \text { Bam HI fragment } \\
\text { containing the } T t h \text { ruvA }^{+} \text {gene }\end{array}$ & This study \\
\hline pTRA126 & $\begin{array}{l}\text { pBluescript II SK(+)derivative carrying } 2.2-\mathrm{kb} \text { Bam HI- } \\
\text { HindIII fragment containing the Tth ruv } A^{+} \text {gene }\end{array}$ & This study \\
\hline pTRA127 & Tth RuvA onverexpression vector derivative of pAF104 & This study \\
\hline pYWH500 & $\begin{array}{l}\text { pSTV28 derivative cloning vector carrying the same } \\
\text { multicloning site as pTF } 100\end{array}$ & This study \\
\hline pYWH501 & pYWH500 derivative carrying the $E$. coli ruv $B^{+}$gene & This study \\
\hline pYWH800 & pYWH500 derivative carrying the Tth ruv $B^{+}$gene & This study \\
\hline
\end{tabular}


pTRA104. The 5.5-kb DNA fragment was digested with several restriction enzymes. The pTRA126 clone was created by ligating the resulting $2.2-\mathrm{kb}$ Bam HI-HindIII fragment containing the Tth ruvA gene to a BamHI-HindIIIdigested pBluescript II SK(+) plasmid. The nucleotide sequence of both strands of pTRA126 was determined using an automated DNA sequencer (Applied Biosystems, model 373XL).

Construction of pTRA127, a Tth RuvA-overproducing plasmid. A pair of DNA primers (F2; 5'-CCAGGGCATATGATCCGCTACCTCCGGGG-3' and R2; 5'GGAGGGGATCCTAGCGGAGGCGCTTTAGGG-3') were used to create an NdeI site at the first ATG codon of the Tth ruvA gene and to create a BamHI site downstream from the stop codon. The underlined portion of each primer indicates the respective $N d e \mathrm{I}$ and BamHI sites. The Tth ruvA gene, which was amplified by PCR using these primers and pTRA126 DNA as a template, was ligated to the NdeI-BamHI site of pAF104, resulting in pTRA127.

Purification of Tth RuvA protein. E. coli HRS4000 harboring pLysS was transformed with pTRA127. The transformants were subsequently grown in 1.5 liters of LB medium containing ampicillin at $37^{\circ} \mathrm{C}$ until an $\mathrm{A}_{600}$ of about 0.6 was achieved. Isopropyl- $\beta$-D-thiogalactopyranoside was then added to a final concentration of $1 \mathrm{mM}$, and the culture was incubated for an additional 4 hours. The cells were collected by centrifugation, resuspended in R-buffer (20 mM Tris- $\mathrm{HCl} \mathrm{pH} 8.0,1 \mathrm{mM}$ EDTA, $7 \mathrm{mM} \beta$-mercaptoethanol, and $10 \%$ glycerol) containing $500 \mathrm{mM} \mathrm{NaCl}$, and disrupted by sonication. To denature most of the endogenous $E$. coli proteins, this crude cell extract was incubated at $75^{\circ} \mathrm{C}$ for $15 \mathrm{~min}$. The cell extract was then centrifuged at $10,000 \times \mathrm{g}$ for 15 min. Ammonium sulfate was gradually added to the supernatant to achieve $40 \%$ saturation. The mixture was stirred for $30 \mathrm{~min}$ and then centrifuged at $27,000 \times \mathrm{g}$ for $15 \mathrm{~min}$. The pellet was resuspended in R-buffer containing $100 \mathrm{mM} \mathrm{NaCl}$ and $2 \mathrm{M}$ urea, and dialyzed against the same buffer overnight. Urea was added to prevent aggregation of Tth RuvA at high concentrations. After the precipitate was removed by centrifugation $(27,000 \times \mathrm{g}$ for $15 \mathrm{~min}$ ), the supernatant was applied to an SPSepharose column (Amersham Pharmacia Biotech) equilibrated with R-buffer containing $100 \mathrm{mM} \mathrm{NaCl}$ and $2 \mathrm{M}$ urea. RuvA was eluted with a 300-ml linear gradient of R-buffer containing $2 \mathrm{M}$ urea plus $100 \mathrm{mM}$ to $1000 \mathrm{mM}$ $\mathrm{NaCl}$. The RuvA peak fractions were pooled and dialyzed overnight against R-buffer containing $100 \mathrm{mM} \mathrm{NaCl}$ and $2 \mathrm{M}$ urea. The dialyzed sample was applied on a Heparin column (Amersham Pharmacia Biotech) equilibrated with R-buffer containing $100 \mathrm{mM} \mathrm{NaCl}$ and $2 \mathrm{M}$ urea. RuvA was eluted with a $300-\mathrm{ml}$ linear gradient of R-buffer containing $2 \mathrm{M}$ urea plus $100 \mathrm{mM}$ to $1000 \mathrm{mM} \mathrm{NaCl}$. The
RuvA peak fractions were pooled and then applied to a Hiroad 26/60 Sephacryl S-300 HR gel filtration column (Amersham Pharmacia Biotech) equilibrated with R-buffer containing $500 \mathrm{mM} \mathrm{NaCl}$ and $2 \mathrm{M}$ urea. The resulting RuvA peak fractions were pooled and dialyzed against R-buffer containing $100 \mathrm{mM} \mathrm{NaCl}$ and $2 \mathrm{M}$ urea. The dialyzed sample was applied to another Heparin column equilibrated with R-buffer containing $100 \mathrm{mM}$ $\mathrm{NaCl}$ and $2 \mathrm{M}$ urea. The RuvA was eluted with a 300-ml linear gradient of R-buffer containing $2 \mathrm{M}$ urea plus 100 $\mathrm{mM}$ to $1000 \mathrm{mM} \mathrm{NaCl}$. The RuvA peak fractions were pooled and dialyzed against R-buffer containing $500 \mathrm{mM}$ $\mathrm{NaCl}$. The protein concentration was determined using an extinction coefficient of $\varepsilon 280=2600 \mathrm{M}^{-1} \mathrm{~cm}^{-1}$.

Tth RuvB and E. coli RuvA and RuvB proteins. Tth RuvB and E. coli RuvA and RuvB proteins were purified as described previously (Hishida et al., 1999; Nishino et al., 1998; Yamada et al., 1999).

Computer analysis. A multiple sequence alignment of RuvA orthologs was constructed with the CLUSTAL W program (Thompson et al., 1994) and was modified by visual inspection to increase the similarity. A phylogenetic tree was constructed using the neighbor-joining method (Saitou and Nei, 1987).

Construction of synthetic Holliday junctions. A synthetic Holliday junction was constructed by annealing four 72mer oligonucleotides, JY-11, JY-12, JY-13 and JY14, as described previously (Hishida et al., 1999). Duplex DNA used for a control was constructed by annealing two complementary 72mer oligonucleotides, JY-11 and JY-15 (5'-CAGCGCTTGGTAAACACATAGAATTCTGCTCGGTCTGAGCCGATTGAAGCTTCTCGAGGTTCCTGTC GCTCG-3').

DNA binding assay. Specific binding of RuvA protein to a synthetic Holliday junction labeled with ${ }^{32} \mathrm{P}$ was assessed with a gel mobility retardation assay. A standard reaction mixture $(20 \mu \mathrm{l})$ containing $10 \mathrm{nM}$ synthetic ${ }^{32} \mathrm{P}-$ Holliday junction DNA and Tth RuvA protein in a buffer of $20 \mathrm{mM}$ HEPES-NaOH ( $\mathrm{pH}$ 7.6), $1 \mathrm{mM}$ DTT and 0.01\% bovine serum albumin was incubated for $30 \mathrm{~min}$ on ice. The concentrations of RuvA in the reaction mixture were systematically varied between 0 and $200 \mathrm{nM}$. After incubation, the reaction mixtures were analyzed by polyacrylamide gel electrophoresis (PAGE) on 6\% polyacrylamide gels. The protein-DNA complexes were visualized with a Fuji BAS1500 image analyzer. The same procedure was carried out for the control in which $10 \mathrm{nM}{ }^{32} \mathrm{P}$ duplex DNA was used in the reaction mixture in place of the Holliday junction DNA.

Branch migration assay. The branch migration activ- 
ity of RuvB was assayed using the synthetic Holliday junction essentially as described previously (Yamada et al., 1999). Reaction mixtures containing one of the following pairings: 1) E. coli RuvA and E. coli RuvB, 2) Tth RuvA and E. coli RuvB, 3) E. coli RuvA and Tth RuvB, or 4) Tth RuvA and Tth RuvB, were prepared. The standard reaction mixture $(20 \mu \mathrm{l})$ contained $5 \mathrm{nM}$ synthetic ${ }^{32} \mathrm{P}$-Holliday junction DNA, $150 \mathrm{nM}$ RuvB and varying concentrations of RuvA ( 0 to $200 \mathrm{nM}$ ) in a buffer of $20 \mathrm{mM}$ HEPES-NaOH (pH 7.6), $1 \mathrm{mM}$ DTT, $5 \mathrm{mM} \mathrm{MgCl}_{2}$, and $20 \%$ glycerol. After incubation for $10 \mathrm{~min}$ or $30 \mathrm{~min}$ at either $37^{\circ} \mathrm{C}$ or $55^{\circ} \mathrm{C}$, the reaction products were analyzed by $9 \%$ PAGE.

ATPase assay. The ATP hydrolysis reaction catalyzed by Tth RuvB protein was measured essentially as described previously (Yamada et al., 1999). The standard reaction mixture $(20 \mu \mathrm{l})$ contained $0.67 \mathrm{mM}$ supercoiled pUC19 DNA, $0.2 \mu \mathrm{M}$ RuvB, varying concentrations of RuvA (0 to $2000 \mathrm{nM}$ ), and $2 \mathrm{mM}\left[\gamma_{-}{ }^{32} \mathrm{P}\right]$ ATP in a buffer of $20 \mathrm{mM}$ HEPES-NaOH (pH 7.6), $10 \mathrm{mM} \mathrm{MgCl}_{2}, 10 \%$ glycerol, and $1 \mathrm{mM}$ DTT. DNA concentration is expressed in terms of the nucleotide concentration only in this assay. The hydrolyzed Pi was measured using a Fuji BAS1500 image analyzer.

\section{RESULTS AND DISCUSSION}

Cloning and sequencing of the $T$. thermophilus $\mathrm{HB} 8$ ruvA gene. The DNA fragment containing the ruvA gene was amplified from genomic DNA using primers that corresponded to sequences flanking the Tth ruvA coding region. This fragment was used as a probe to identify restriction fragments carrying the Tth ruvA gene. A 5.5$\mathrm{kb}$ Bam HI genomic fragment was first identified by Southern blot hybridization and was subsequently confirmed to contain the full length of the Tth ruvA gene. This fragment was cloned into the BamHI site of pUC19, thereby producing pTRA104. The restriction enzyme cleavage map of this fragment is shown in Fig. 1.

The 2.2-kb BamHI-HindIII fragment that was expected to contain the entire ruvA gene was subcloned into the pBluescript II SK(+) vector, thereby forming pTRA126. Analysis of the nucleotide sequence of the 2.2-kb insert (DDBJ Accession No. AB048605) revealed three open reading frames corresponding to the putative enoyl-CoA hydratase $\alpha$ subunit gene, the Tth ruvA gene and a putative ysaA homolog of Bacillus subtilis, whose function is unknown (Fig. 1). Tth ruvA encodes a protein of 20,414 Da consisting of 191 amino acid residues. The GC content of Tth ruvA is $70.8 \%$, which is similar to that of Tth ruvB $(70.1 \%)$ (Yamada et al., 1999). In contrast to most prokaryotes, in which ruvA and $\operatorname{ruvB}$ constitute a single operon (Hishida et al., 1996; Sharples et al., 1999), neither $r u v B$ nor ruvC were found in the vicinity of ruvA in

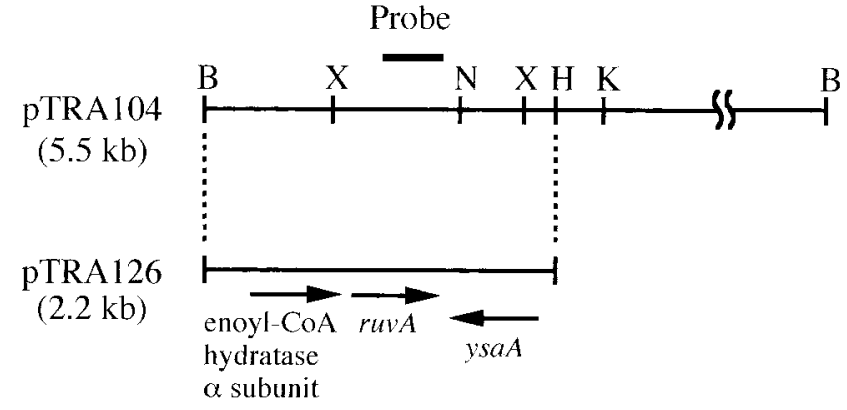

Fig. 1. Physical map of cloned and subcloned DNA fragments containing the ruvA gene from T. thermophilus HB8. The arrows indicate open reading frames. The bold line indicates the probe region used for cloning. Restriction enzyme recognition sites: B, BamHI; H, HindIII; K, KpnI; N, NcoI; X, XhoI.

\section{T. thermophilus HB8.}

Multiple sequence alignment and phylogenetic analysis. The amino acid sequence of Tth RuvA was aligned with the sequences of other bacterial RuvA proteins (Fig. 2). X-ray crystallography of E. coli RuvA revealed that it consists of three domains (Nishino et al., 1998; Rafferty et al., 1996) and the secondary structure of $E$. coli RuvA is shown above the alignment (Fig. 2). The deduced sequence of Tth RuvA is 37\% identical and $75.4 \%$ similar to the sequence of $E$. coli RuvA. The deduced sequence of Tth RuvB is $56 \%$ identical to that of $E$. coli RuvB (Yamada et al., 1999). This is in accord with the observation that the sequence conservation among RuvA orthologs is generally much lower than that among RuvB orthologs (Hishida et al., 1996; Sharples et al., 1999). Notably, Tth RuvA, as well as RuvAs from Mycoplasma pneumoniae (Mpn), Mycoplasma genitalium (Mge), Helicobacter pylori (Hpy) and Borrelia burgdorferi (Bbu), have a much shorter linker region between the $\alpha 6$ and $\alpha 7$ helices than $E$. coli RuvA.

Using the alanine scanning mutagenesis technique, we previously identified six functionally important amino acid residues in $E$. coli RuvA. That study revealed that mutations of Ile-2, Leu-110, Leu-167, Leu-170, Tyr-172 and Leu-199 resulted in loss of RuvA function in vivo (Nishino et al., 1998 and T. Fujita, H. I. and H. S. unpublished data). Four of these amino acids (Leu-167,-170, and -199 and Tyr-172) are hydrophobic residues located in domain III of $E$. coli RuvA and are likely to form an interface with RuvB. X-ray crystallography of the $E$. coli RuvA-Holliday junction complex revealed that two helix-hairpin-helix $(\mathrm{HhH})$ motifs in domain II are involved in Holliday junction binding (Ariyoshi et al., 2000). The nitrogens of the main chain amides of Gly-80 and Gly-82 in the hairpin in $\mathrm{HhH}$ I interact with phosphate oxygens of the junction DNA through hydrogen bonds. A similar interaction occurs for the amides of Gly-115 and Gly-117 in the hairpin in HhH II. The side chains of Lys-84 in the second 

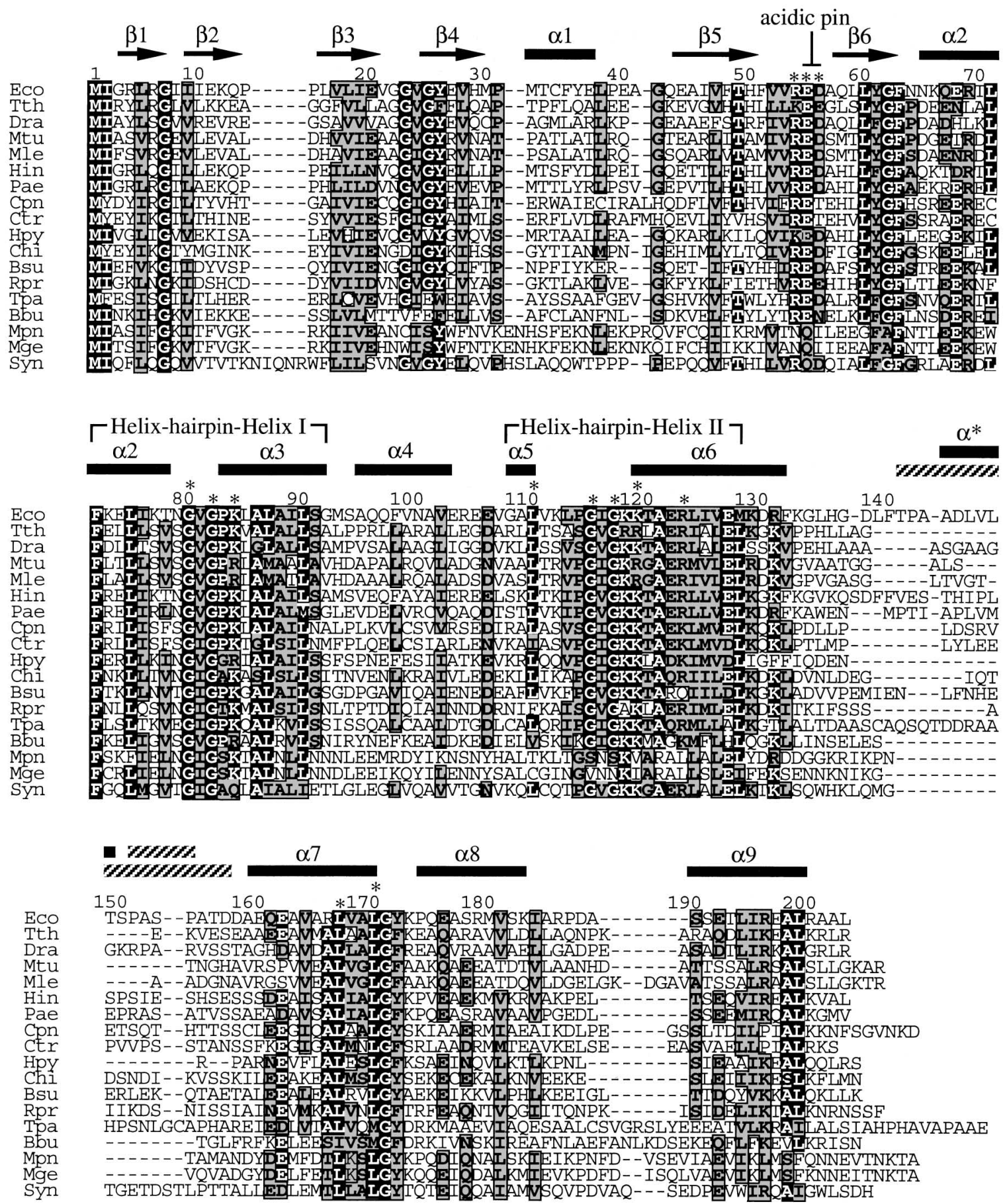

Fig. 2. Sequence alignment of Tth RuvA and other bacterial orthologs. Amino acid sequences of various RuvAs from Escherichia coli (Eco), Thermus thermophilus HB8 (Tth), Deinococcus radiodurans (Dra), Mycobacterium tuberculosis (Mtu), Mycobacterium leprae (Mle), Haemophilus influenzae (Hin), Pseudomonas aeruginosa (Pae), Chlamydia pneumoniae (Cpn), Chlamydia trachomatis (Ctr), Helicobacter pylori (Hpy), Clostridium histolyticum (Chi), Bacillus subtilis (Bsu), Rickettsia prowazekii (Rpr), Treponema pallidum (Tpa), Borrelia burgdorferi (Bbu), Mycoplasma pneumoniae (Mpn), Mycoplasma genitalium (Mge), and Synechocystis sp (Syn) were aligned using the CLUSTAL W multiple sequence alignment program (Thompson et al., 1994). Amino acid positions that contained more than 12/18 similar amino acids and identical amino acids are shaded in gray and black, respectively. Similar amino acids were defined as follows: LIMV, SPTAG, YWF, DEQN, and KRH. Particularly important amino acid residues that are mentioned in the text are marked by asterisks above the $E$. coli RuvA sequence. The secondary structure of $E$. coli RuvA ( $\alpha$ helices and $\beta$ strands) is shown above the alignment and is indicated by arrows ( $\beta$ strands) and bold lines ( $\alpha$ helices). Domains 1,2 , and 3 include $\beta 1$ to $\beta 6, \alpha 2$ to $\alpha 6$, and $\alpha 7$ to $\alpha 9$, respectively (Ariyoshi et al., 2000; Nishino et al., 1998; Rafferty et al., 1996). The striped bars between domains 2 and 3 indicate disordered structure. The entire linker region (residues 141-157) adopted a disordered structure in the DNA-free crystal, while the region contained a short a helix $\left(\alpha^{*}\right)$ (residues 144-149) in the complex with the synthetic Holliday junction (Ariyoshi et al., 2000). 


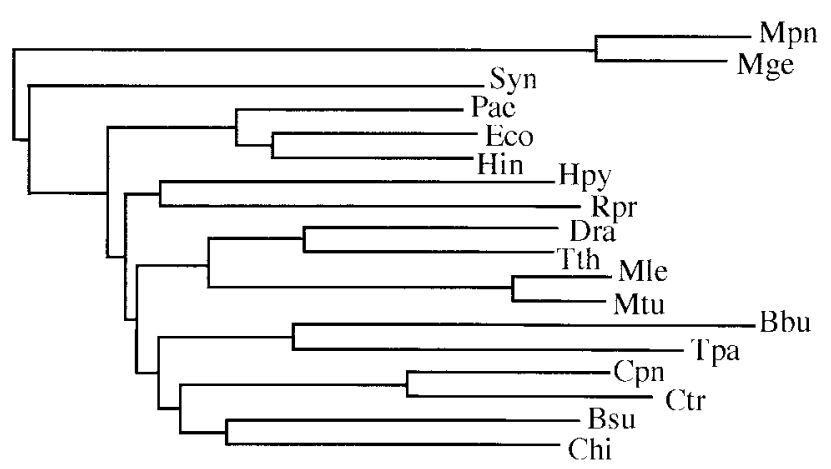

0.1

Fig. 3. The unrooted phylogenetic tree of 18 bacterial RuvA orthologs. Based on the multiple alignment shown in Fig. 2, a phylogenetic tree was constructed using the neighbor-joining method (Saitou and Nei, 1987). Abbreviations of the species are the same as those in Fig. 2.

(A)

\begin{tabular}{|c|c|c|}
\hline $\begin{array}{c}\Delta r u v A \text { strair } \\
+ \\
\text { plasmid }\end{array}$ & no UV & $\mathrm{UV} 30 \mathrm{~J} / \mathrm{m}^{2}$ \\
\hline & & \\
\hline
\end{tabular}

(B)

$$
\begin{gathered}
\Delta r u v A B \text { strain } \\
+ \\
\text { plasmid }
\end{gathered}
$$

\begin{tabular}{|c|c|c|}
\hline ruvA & $\operatorname{ruvB} B$ & no UV \\
\hline & & 020 \\
\hline E. coli & $F \bar{c}$ i & 00 \\
\hline Tth & - & 0000 \\
\hline - & Tth & 10000 \\
\hline $\begin{array}{l}\text { E. coli } \\
\text { Tth }\end{array}$ & E. coli & $\begin{array}{l}0000 \\
0000\end{array}$ \\
\hline E. coli & Tth & 000 . \\
\hline Tth & Tth & 000 \\
\hline
\end{tabular}

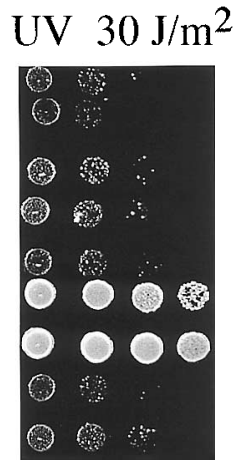

Fig. 4. In vivo complementation assay. Serial dilutions of cells were spotted on LB plates, followed by UV irradiation. The plates were incubated at $37^{\circ} \mathrm{C}$ for about $15 \mathrm{hr}$. (A) The UV sensitivity of an E. coli ruvA mutant strain transformed with either pAF104 (vector), pAF134 (E. coli ruvA), or pTRA127 (Tth $\operatorname{ruvA}$ ). (B) The sensitivity of an $E$. coli ruvA ruvB mutant strain carrying both homologous and heterologous combinations of ruvA and ruvB genes. The plasmids carrying ruvA were pAF134 (E. coli ruvA) and pTRA127 (Tth ruvA), both of which are derivatives of vector plasmid pAF104. The plasmids carrying $r u v B$ were pYWH501 (E. coli ruvB) and pYWH800 (Tth ruvB), both of which are derivatives of vector plasmid pYWH500. The plasmids carrying the $\operatorname{ruv} A$ or $\operatorname{ruvB}$ genes, respectively, were compatible in the strain. Bars indicate the empty vector. helix in HhH I and those of Lys-119 and Arg-123 in the second helix in HhH II also interact with the junction DNA. The observation that the mutant protein (Leu-110 to Ala) does not bind junction DNA in vitro (Nishino et al., 1998), suggests that this mutation may cause structural disorder of the functional HhH II motif. The side chains of Arg-54, Glu-55 and Asp-56, the latter two of which are collectively called the "acidic pin" (Rafferty et al., 1996), are located in the vicinity of the junction center. They are involved in the disruption of base-pairing at the junction center, which facilitates RuvB-mediated branch migration (Ariyoshi et al., 2000). The amino acid residues corresponding to the important ones revealed by these $E$. coli RuvA studies are also highly conserved in $T t h$ RuvA (Fig. 2).

On the basis of this alignment, we constructed an unrooted molecular phylogenetic tree of RuvA orthologs using the neighbor-joining method (Fig. 3). Tth RuvA is evolutionarily most related to Deinococcus radiodurans RuvA. The phylogenetic relationships among RuvA orthologs are similar to those among RuvB orthologs and among 16S rRNA sequences (Maidak et al., 1999). This observation may suggest that RuvA, as well as RuvB, evolved from the earliest times as an important protein with a fundamental cellular function, like DNA and RNA polymerases and many enzymes involved in translation. Genetic studies show that RuvA, RuvB, and RuvC proteins work together in sexual conjugation and recombinational DNA repair in E. coli (Mandal et al., 1993; Takahagi et

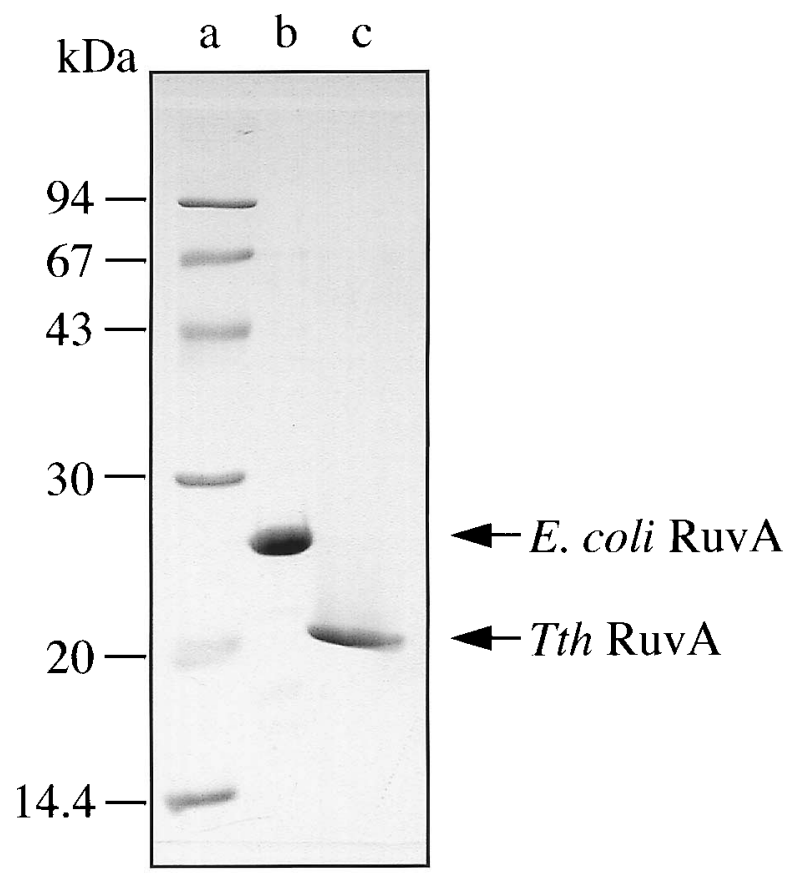

Fig. 5. SDS-PAGE analysis of Tth RuvA. Proteins were separated by $15 \%$ SDS-PAGE and stained with Coomassie brilliant blue. Lanes: a, molecular weight markers; b, purified E. coli RuvA; c, purified Tth RuvA. 
al., 1991). The fact that RuvA and RuvB are widespread throughout bacterial species whereas RuvC is less common (Sharples et al., 1999), suggests that an alternative Holliday junction resolvase or resolution mechanism may exist in certain bacteria that lack RuvC. The possibility has been suggested that RuvAB, independently of RuvC, is involved in the restoration of stalled replication forks (Seigneur et al., 1998), and this may also account for the higher conservation of RuvAB than RuvC.
The Tth ruvA gene complements the DNA repair defect of $\boldsymbol{E}$. coli ruvA mutants. To examine whether the Tth ruvA gene could substitute for the recombination repair function of the $E$. coli ruvA gene, a plasmid carrying the Tth ruvA gene (pTRA127) was introduced into the E. coli $\triangle r u v A$ strain (HRS2300) by transformation, and the UV sensitivity of the transformant was compared with that of the control strain (Fig. 4A). The results indicated that the Tth ruvA gene was indeed able to complement

(A)

Tth RuvA (nM) $\quad 0 \quad 1 \quad 5 \quad 10 \quad 2550100200 \quad 02000$

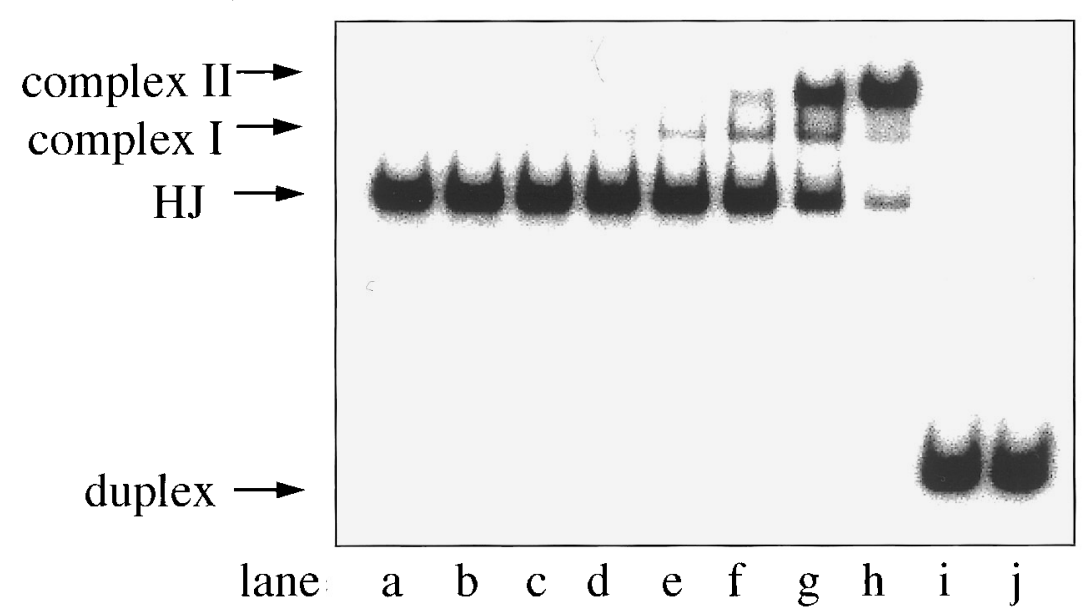

(B)

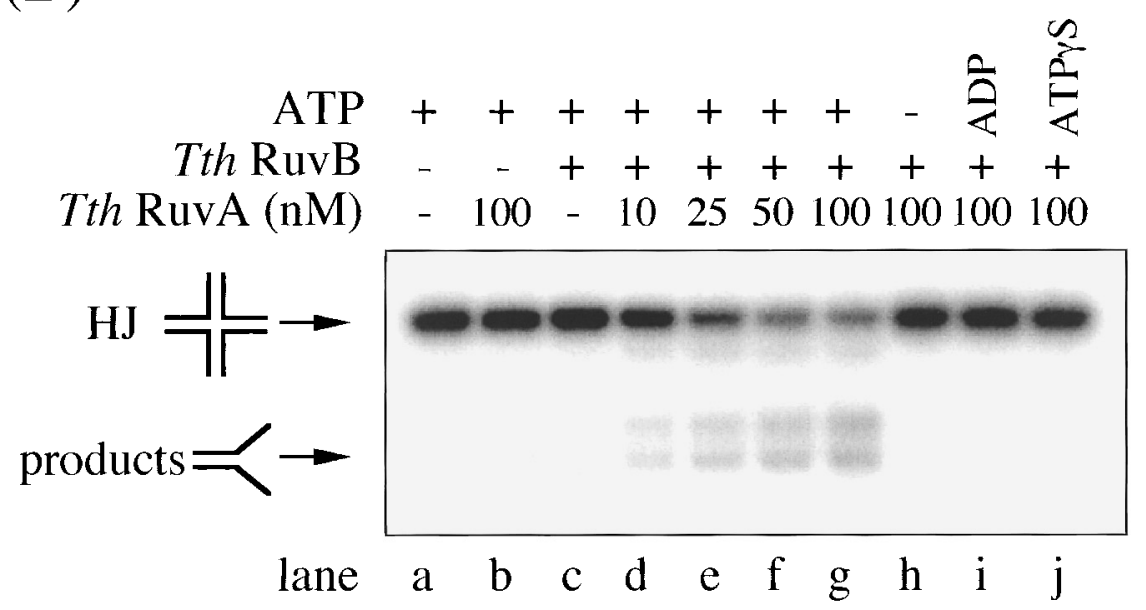

Fig. 6. (A) DNA-binding activity of Tth RuvA was analyzed by a gel retardation assay. Reactions were carried out with either $10 \mathrm{nM}$ junction DNA (synthetic Holliday junction, lanes a to h) or duplex DNA (lanes i and j) and the indicated amount of Tth RuvA protein. The protein-DNA complexes were analyzed by 6\% PAGE. (B) RuvA-induced stimulation of the branch migration activity of $T t h$ RuvB for the synthetic Holliday junctions. Five nanomolar ${ }^{32} \mathrm{P}$-labeled junction was incubated with $1 \mu \mathrm{M}$ Tth RuvB at $55^{\circ} \mathrm{C}$ either in the absence of Tth RuvA (lane c) or in the presence of different concentrations of Tth RuvA (lanes d to j). Following deproteinization, the products were analyzed by $9 \%$ PAGE. ATP was added to the reaction mixtures loaded into lanes a to g. No ATP was present in the sample in lane h. ADP (lane i) and ATP $\gamma \mathrm{S}$ (lane j) were substituted for ATP in the respective reaction mixtures. 
the repair defect of the E. coli ruvA mutant. The repair capability of the Tth ruvA gene, however, was less efficient than that of the E. coli ruvA gene.

To examine the functional interactions between heterologous combinations of RuvA and RuvB in vivo, an E. coli $\triangle r u v A B$ strain was transformed with different combinations of the $\operatorname{ruv} A B$ genes, and the UV sensitivities of the transformants were compared (Fig. 4B). The combination of the Tth ruvA (pTRA127) and E. coli ruvB (pYWH501) genes complemented the $E$. coli $\triangle r u v A B$ strain (HRS3403) as efficiently as the intrinsic combination of the E. coli ruvA (pAF134) and E. coli ruvB (pYWH501) genes. Inefficient restoration of DNA repair by Tth ruvA in the $\triangle r u v A$ strain (HRS2300) is probably due to lower expression of $E$. coli RuvB from the chromosome than from the multicopy plasmid. Although $E$. coli ruvA complemented the UV sensitivity of the $\triangle r u v A$ strain, the expression level of $r u v B$ was reduced compared to that in the wild-type strain after the cells were irradiated with UV at $60 \mathrm{~J} / \mathrm{m}^{2}$, due to a polar effect of the insertion mutation (data not shown). A higher amount of $E$. coli RuvB may be required to form a fully functional complex with $T t h$ RuvA than with $E$. coli RuvA.

On the other hand, consistent with our previous findings that Tth ruvB does not complement the repair deficiency of an E. coli $\Delta r u v B$ strain (Yamada et al., 1999), neither combinations of $E$. coli ruvA (pAF134) and Tth ruvB (pYWH800) nor Tth ruvA (pTRA127) and Tth ruvB (pYWH800) restored the UV sensitivity of the $E$. coli $\triangle r u v A B$ strain. Possible explanations for these results are: 1) Tth RuvB may not function well at the optimum growing temperature for $E$. coli $\left(37^{\circ} \mathrm{C}\right)$, a temperature which is much lower than the optimum growing temperatures of the thermophilic bacteria, and/or 2) Tth RuvB may not functionally interact with $E$. coli RuvC in vivo.

Biochemical properties of $T t h$ RuvA protein. Tth RuvA was purified from the T7-overexpression system by successive column chromatography as described in MATERIALS AND METHODS. Tth RuvA preparations showed a single band in SDS-PAGE (Fig. 5). From 1.5 liters of culture, about $10 \mathrm{mg}$ of pure Tth RuvA was obtained. Using this purified protein, we examined the biochemical properties of Tth RuvA in vitro.

First, we examined the DNA-binding activity and specificity of Tth RuvA for four-way junctions (synthetic Holliday junctions). As shown in Fig. 6A, Tth RuvA formed two kinds of complexes with the junction DNA: one that migrated relatively quickly (complex I) and the other that migrated more slowly (complex II) through the gel, as observed in the case of the $E$. coli RuvA-Holliday junction complex (Parsons et al., 1992). Complex I corresponds to the junctions bound by one RuvA tetramer, and Complex II corresponds to junctions bound by two RuvA tetramers. Tth RuvA did not bind to duplex DNA, even when RuvA was present at a concentration 200 times higher than that of duplex DNA. This observation clearly demonstrates that Tth RuvA has a highly specific affinity for junction DNA, as shown previously for its $E$. coli ortholog (Iwasaki et al., 1992; Parsons et al., 1992).

We next examined the ability of Tth RuvA to promote RuvB-mediated branch migration of Holliday junctions. To avoid spontaneous branch migration of the synthetic Holliday junction, all reactions were carried out at $55^{\circ} \mathrm{C}$, a temperature slightly lower than the optimal growing temperature $\left(\sim 70^{\circ} \mathrm{C}\right)$ of $T$. thermophilus HB8. As shown in Fig. 6B, the Tth RuvA-RuvB complex promoted branch migration in a manner that was dependent on the concentration of RuvA present in the reaction mixture. The reaction was also dependent on ATP hydrolysis because neither ADP nor non-hydrolyzable ATP $\gamma \mathrm{S}$ was able to promote Tth RuvA-RuvB-associated branch migration.

E. coli RuvB has an intrinsic ATPase activity, which is greatly stimulated by RuvA and duplex DNA, and particularly by circular duplex DNA (Marrione and Cox, 1996; Shiba et al., 1991). The ATPase activity of Tth RuvB in the presence of Tth RuvA displayed essentially the same characteristics as that of $E$. coli RuvB (Fig. 7).

The branch migration activities of heterologous combinations of Tth and E. coli proteins were examined (Fig. 8). Tth RuvA stimulated the branch migration activities of

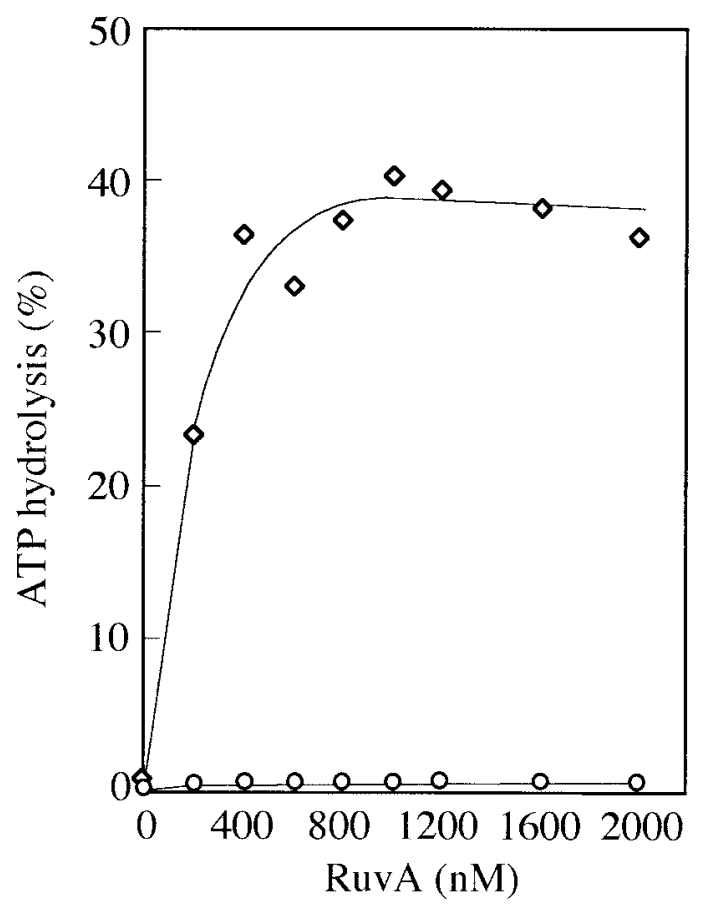

Fig. 7. Stimulation of the ATPase activity of Tth RuvB by Tth RuvA. The ATPase activity of Tth RuvB (200 nM) in the presence of Tth RuvA at the indicated concentration were measured in the presence (squares) and absence (circles) of dsDNA under the standard conditions at $70^{\circ} \mathrm{C}$ for $10 \mathrm{~min}$. Each point represents the average of four independent experiments. 


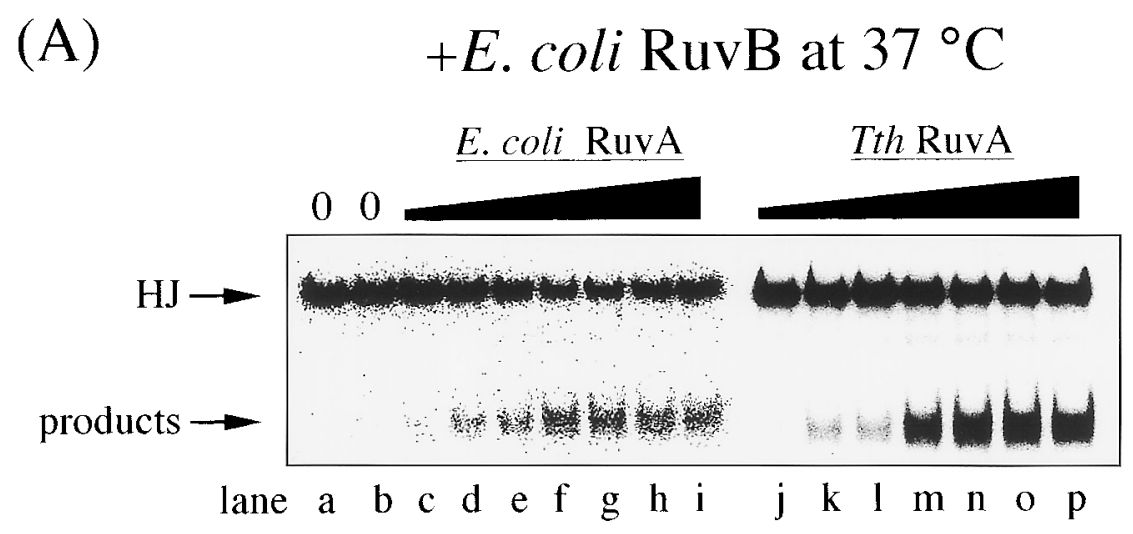

(B)
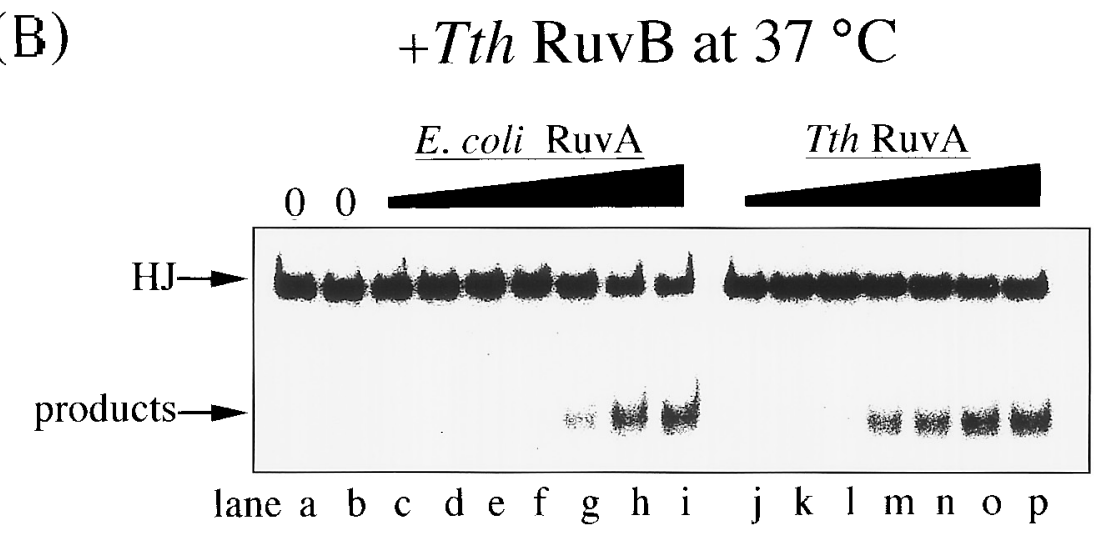

(C)

+ Tth RuvB at $55^{\circ} \mathrm{C}$

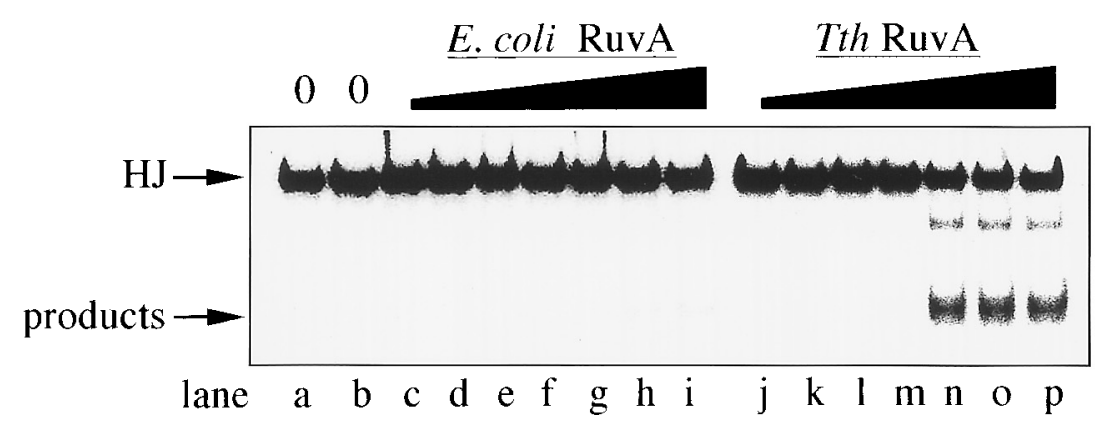

Fig. 8. Effects of RuvA on branch migration activity of RuvB in combination with E. coli and T. thermophilus HB8 proteins. (A) Effects of $E$. coli and Tth RuvA proteins on the branch migration activity of $E$. coli RuvB at $37^{\circ} \mathrm{C}$. $\quad E$. coli RuvB ( $\left.150 \mathrm{nM}\right)$ and various concentrations of $E$. coli RuvA (lanes b to i) or Tth RuvA (lanes j to p) were added to reaction mixtures containing $5 \mathrm{nM}{ }^{32} \mathrm{P}$-labeled junction and incubated for $10 \mathrm{~min}$ at $37^{\circ} \mathrm{C}$. (B) Effects of E. coli and Tth RuvA on the branch migration activity of Tth RuvB at $37^{\circ} \mathrm{C}$. Tth RuvB (150 nM) and various concentrations of $E$. coli RuvA (lanes b to i) or Tth RuvA (lanes j to p) were added to reaction mixtures containing $5 \mathrm{nM}{ }^{32} \mathrm{P}$-labeled junction and incubated for $10 \mathrm{~min}$ at $37^{\circ} \mathrm{C}$. (C) Effects of $E$. coli and Tth RuvA proteins on the branch migration activity of $T t h$ RuvB at $55^{\circ} \mathrm{C}$. The reactions were carried out under the same conditions as in (B) except that the reaction mixtures were incubated at $55^{\circ} \mathrm{C}$. Protein concentrations: Lane a, no RuvA or RuvB; lane b, no RuvA; lanes c and j, 1 nM RuvA; lanes $\mathrm{d}$ and k, $5 \mathrm{nM}$ RuvA; lanes e and l, $10 \mathrm{nM}$ RuvA; lanes f and m, $25 \mathrm{nM}$ RuvA; lanes g and n, $50 \mathrm{nM}$ RuvA; lanes h and o, $100 \mathrm{nM}$ RuvA; and lanes i and p, $200 \mathrm{nM}$ RuvA. Lanes b to $\mathrm{p}$ also contained $150 \mathrm{nM}$ RuvB. 
RuvB proteins from both $T$. thermophilus $\mathrm{HB} 8$ and $E$. coli nearly equally (Figs. 8A and 8B). At $37^{\circ} \mathrm{C}, E$. coli RuvA stimulated the activity of Tth RuvB less efficiently (Fig. 8B). These results are consistent with data from our in vivo complementation experiments: Tth ruvA complemented the UV repair defect of the $E$. coli $\Delta r u v A$ mutant, whereas $T t h$ ruvB did not complement this defect of the $E$. coli $\Delta r u v B$ mutant (Fig. 4). At $55^{\circ} \mathrm{C}$, which was closer to the optimal physiological temperature for T. thermophilus HB8 than $37^{\circ} \mathrm{C}$, Tth RuvA promoted the branch migration of Tth RuvB, while E. coli RuvA did not (Fig. 8C). However, the reaction efficiency of branch migration promoted by the Tth RuvAB complex was almost the same at both $37^{\circ}$ and $55^{\circ} \mathrm{C}$ (compare Fig. $8 \mathrm{C}$ lanes $\mathrm{j}$ - p with Fig. 8B lanes j - p) although a larger amount of products would be expected at $55^{\circ} \mathrm{C}$. Essentially the same results were reproducibly obtained from several independent experiments. We cannot explain this discrepancy at present.

To evaluate the heat stability of Tth RuvA, we performed two assays using $E$. coli RuvA as a control. After RuvA proteins were incubated at $90^{\circ} \mathrm{C}$ for $1 \mathrm{hr}$, we examined whether they retained the specific ability of Holliday junction binding by the gel shift assay. Both E. coli RuvA and Tth RuvA retained full junction-binding activity (data not shown). Similarly, after pre-incubation at $90^{\circ} \mathrm{C}$ for 1 $\mathrm{hr}$, the ability of RuvA to promote branch migration in association with $E$. coli RuvB was examined at $37^{\circ} \mathrm{C}$. The results showed that neither protein exhibited any detectable loss of activity (data not shown). Thus, we conclude that Tth RuvA, and surprisingly E. coli RuvA as well, are highly heat stable, while Tth RuvB is more stable than E. coli RuvB in terms of its ATPase and branch migration activities (Yamada et al., 1999). Therefore, the fact that the hetero-complex between $E$. coli RuvA and Tth RuvB did not promote branch migration at $55^{\circ} \mathrm{C}$ (Fig. $8 \mathrm{C}$ ) cannot be explained simply by the heat lability of $E$. coli RuvA. It is possible that the complex formation is labile because of non-optimal interfaces between the RuvA and RuvB from different bacterial sources.

Conclusions and perspectives. In this study, we cloned the ruvA gene from T. thermophilus HB8 and analyzed its sequence. Its deduced primary amino acid sequence is $37 \%$ identical to that of $E$. coli RuvA. Although this homology is not remarkably high, nearly all the amino acid residues important for RuvA functions, which have been revealed so far by mutational and structural analyses of $E$. coli RuvA, are well conserved in Tth RuvA. Tth ruvA complements the UV sensitivity of $E$. coli $\triangle r u v A$ strains. In contrast, Tth ruvB does not complement the UV sensitivity of $E$. coli $\Delta r u v B$ strains. Tth RuvA protein exhibits biochemical activities very similar to those exhibited by $E$. coli RuvA. In addition, Tth RuvA stimulates the promotion of branch migration by $E$. coli RuvB in vitro, which is well consistent with the results of in vivo hetero-complementation experiments.

It is widely believed that proteins from thermophilic organisms are more stable and give rise to superior crystals for X-ray diffraction analysis than the corresponding proteins from mesothermic organisms. Indeed, we have been able to produce Tth RuvB crystals suitable for analysis, while we have failed to produce any $E$. coli RuvB crystals (K. Yamada, H. I., T. O., H. S., and K. Morikawa, unpublished results). Tth RuvA, in combination with the Tth RuvB protein isolated previously (Yamada et al., 1999), now should be excellent material for obtaining crystals of RuvAB proteins in complexes with Holliday junction DNA for X-ray crystallography. This will be critically important for understanding the branch migration mechanism at the atomic level.

We thank D. Dziadkowiec and E. Nakajima for correcting the English in this manuscript. This work was supported by Grantsin-Aids for Specific Research on Priority Areas from the Ministry of Education, Science, Sports and Culture of Japan (08280102 and 0828010) to H. S.

\section{REFERENCES}

Ariyoshi, M., Nishino, T., Iwasaki, H., Shinagawa, H. and Morikawa, K. (2000) Crystal structure of the Holliday junction DNA in complex with a single RuvA tetramer. Proc. Natl. Acad. Sci. USA 97, 8257-8262.

Bachmann, B. J. (1972) Pedigrees of some mutant strains of Escherichia coli K-12. Bacteriol. Rev. 36, 525-557.

Benson, F. E., Illing, G. T., Sharples, G. J. and Lloyd, R. G. (1988) Nucleotide sequencing of the ruv region of Escherichia coli K-12 reveals a LexA regulated operon encoding two genes. Nucleic Acids Res. 16, 1541-1549.

Dunderdale, H. J., Benson, F. E., Parsons, C. A., Sharples, G. J., Lloyd, R. G. and West, S. C. (1991) Formation and resolution of recombination intermediates by $E$. coli RecA and RuvC proteins. Nature 354, 506-510.

Guzman, L. M., Belin, D., Carson, M. J. and Beckwith, J. S. (1995) Tight regulation, modulation, and high-level expression by vectors containing the arabinose $\mathrm{P}_{\mathrm{BAD}}$ promoter. J. Bacteriol. 177, 4121-4130.

Hishida, T., Iwasaki, H., Ishioka, K. and Shinagawa, H. (1996) Molecular analysis of the Pseudomonas aeruginosa genes, $\operatorname{ruv} A, \operatorname{ruv} B$ and $r u v C$, involved in processing of homologous recombination intermediates. Gene 182, 63-70.

Hishida, T., Iwasaki, H., Yagi, T. and Shinagawa, H. (1999) Role of Walker Motif A of RuvB protein in promoting branch migration of Holliday junctions. Walker motif a mutations affect ATP binding, ATP hydrolyzing, and DNA binding activities of RuvB. J. Biol. Chem. 274, 25335-25342.

Ishioka, K., Fukuoh, A., Iwasaki, H., Nakata, A. and Shinagawa, H. (1998) Abortive recombination in Escherichia coli ruv mutants blocks chromosome partitioning. Genes Cells 3, 209-220.

Iwasaki, H., Shiba, T., Makino, K., Nakata, A. and Shinagawa, H. (1989) Overproduction, purification, and ATPase activity of the Escherichia coli RuvB protein involved in DNA repair. J. Bacteriol. 171, 5276-5280.

Iwasaki, H., Takahagi, M., Nakata, A. and Shinagawa, H. (1992) Escherichia coli RuvA and RuvB proteins specifically inter- 
act with Holliday junctions and promote branch migration. Genes Dev. 6, 2214-2220.

Iwasaki, H., Takahagi, M., Shiba, T., Nakata, A. and Shinagawa, H. (1991) Escherichia coli RuvC protein is an endonuclease that resolves the Holliday structure. EMBO J. 10, 43814389.

Kowalczykowski, S. C., Dixon, D. A., Eggleston, A. K., Lauder, S. D. and Rehrauer, W. M. (1994) Biochemistry of homologous recombination in Escherichia coli. Microbiol. Rev. 58, 401465.

Maidak, B. L., Cole, J. R., Parker, C. T. J., Garrity, G. M., Larsen, N., Li, B., Lilburn, T. G., McCaughey, M. J., Olsen, G. J., Overbeek, R., Pramanik, S., Schmidt, T. M., Tiedje, J. M. and Woese, C. R. (1999) A new version of the RDP (Ribosomal Database Project). Nucleic Acids Res. 27, 171-173.

Mandal, T. N., Mahdi, A. A., Sharples, G. J. and Lloyd, R. G. (1993) Resolution of Holliday intermediates in recombination and DNA repair: indirect suppression of $\operatorname{ruvA}, \operatorname{ruvB}$, and $\operatorname{ruvC}$ mutations. J. Bacteriol. 175, 4325-4334.

Marrione, P. E. and Cox, M. M. (1996) Allosteric effects of RuvA protein, ATP, and DNA on RuvB protein-mediated ATP hydrolysis. Biochemistry 35, 11228-11238.

Nishino, T., Ariyoshi, M., Iwasaki, H., Shinagawa, H. and Morikawa, K. (1998) Functional analyses of the domain structure in the Holliday junction binding protein RuvA. Structure 6, 11-21.

Parsons, C. A., Tsaneva, I., Lloyd, R. G. and West, S. C. (1992) Interaction of Escherichia coli RuvA and RuvB proteins with synthetic Holliday junctions. Proc. Natl. Acad. Sci. USA 89, 5452-5456.

Rafferty, J. B., Sedelnikova, S. E., Hargreaves, D., Artymiuk, P. J., Baker, P. J., Sharples, G. J., Mahdi, A. A., Lloyd, R. G. and Rice, D. W. (1996) Crystal structure of DNA recombination protein RuvA and a model for its binding to the Holliday junction. Science 274, 415-421.

Saitou, N. and Nei, M. (1987) The neighbor-joining method: a new method for reconstructing phylogenetic trees. Mol. Evol. Biol 4, 406-425.

Seigneur, M., Bidnenko, V., Ehrlich, S. D. and Michel, B. (1998) RuvAB acts at arrested replication forks. Cell 95, 419-430.

Shah, R., Bennett, R. J. and West, S. C. (1994) Genetic recombination in E. coli: RuvC protein cleaves Holliday junctions at resolution hotspots in vitro. Cell 79, 853-864.

Sharples, G. J., Ingleston, S. M. and Lloyd, R. G. (1999) Holliday junction processing in bacteria: insights from the evolutionary conservation of RuvABC, RecG, and RusA. J. Bacteriol. 181, 5543-5550.

Sharples, G. J. and Lloyd, R. G. (1991) Resolution of Holliday junctions in Escherichia coli: identification of the ruvC gene product as a 19-kilodalton protein. J. Bacteriol. 173, 7711-7715.
Shiba, T., Iwasaki, H., Nakata, A. and Shinagawa, H. (1991) SOSinducible DNA repair proteins, RuvA and RuvB, of Escherichia coli: Functional interactions between RuvA and RuvB for ATP hydrolysis and renaturation of the cruciform structure in supercoiled DNA. Proc. Natl. Acad. Sci. USA 88, 84458449.

Shiba, T., Iwasaki, H., Nakata, A. and Shinagawa, H. (1993) Escherichia coli RuvA and RuvB proteins involved in recombination repair: physical properties and interactions with DNA. Mol. Gen. Genet. 237, 395-399.

Shida, T., Iwasaki, H., Saito, A., Kyogoku, Y. and Shinagawa, H. (1996) Analysis of substrate specificity of the RuvC Holliday junction resolvase with synthetic Holliday junction. J. Biol. Chem. 42, 26105-26109.

Shinagawa, H. and Iwasaki, H. (1996) Processing the Holliday junction in homologous recombination. Trends Biochem. Sci. 21, 107-111.

Shinagawa, H., Makino, K., Amemura, M., Kimura, S., Iwasaki, H. and Nakata, A. (1988) Structure and regulation of the Escherichia coli ruv operon involved in DNA repair and recombination. J. Bacteriol 170, 4322-9.

Stasiak, A., Tsaneva, I. R., West, S. C., Benson, C. J., Yu, X. and Egelman, E. H. (1994) The Escherichia coli RuvB branch migration protein forms double hexameric rings around DNA. Proc. Natl. Acad. Sci. USA 91, 7618-7622.

Studier, F. W., Rosenberg, A. H., Dunn, J. J. and Dubendorff, J. W. (1990) Use of T7 RNA polymerase to direct expression of cloned genes. Methods Enzymol. 185, 60-89.

Takahagi, M., Iwasaki, H., Nakata, A. and Shinagawa, H. (1991) Molecular analysis of the Escherichia coli ruvC gene, which encodes a Holliday junction-specific endonuclease. J. Bacteriol. 173, 5747-5753.

Thompson, J. D., Higgins, D. G. and Gibson, T. J. (1994) CLUSTAL $\mathrm{W}$ : improving the sensitivity of progressive multiple sequence alignment through sequence weighting, position-specific gap penalties and weight matrix choice. Nucleic Acids Res. 22, 4673-4680.

Tong, J. and Wetmur, J. G. (1996) Cloning, sequencing, and expression of $r u v B$ and characterization of RuvB proteins from two distantly related thermophilic eubacteria. J. Bacteriol. 178, 2695-2700.

Tsaneva, I. R., Müller, B. and West, S. C. (1992) ATP-dependent branch migration of Holliday junctions promoted by the RuvA and RuvB proteins of E. coli. Cell 69, 1171-1180.

West, S. C. (1997) Processing of recombination intermediates by the RuvABC proteins. Annu. Rev. Genet. 31, 213-244.

Yamada, K., Fukuoh, A., Iwasaki, H. and Shinagawa, H. (1999) Novel properties of the Thermus thermophilus RuvB protein, which promotes branch migration of Holliday junctions. Mol. Gen. Genet. 261, 1001-1011. 